\title{
PREDICTION OF THE DETECTION LIMIT IN A NEW COUNTING EXPERIMENT
}

\author{
KWANG-IL SEON \\ Korea Astronomy and Space Science Institute, 61-1 Hwaam-dong Yuseong-gu Daejeon, 305-348, Korea \\ E-mail: kiseon@kasi.re.kr \\ (Received November 16, 200\%; Accepted August 12, 2008)
}

\begin{abstract}
When a new counting experiment is proposed, it is crucial to predict whether the desired source signal will be detected, or how much observation time is required in order to detect the signal at a certain significance level. The concept of the a priori prediction of the detection limit in a newly proposed experiment should be distinguished from the a posteriori claim or decision whether a source signal was detected in an experiment already performed, and the calculation of statistical significance of a measured source signal. We formulate precise definitions of these concepts based on the statistical theory of hypothesis testing, and derive an approximate formula to estimate quickly the a priori detection limit of expected Poissonian source signals. A more accurate algorithm for calculating the detection limits in a counting experiment is also proposed. The formula and the proposed algorithm may be used for the estimation of required integration or observation time in proposals of new experiments. Applications include the calculation of integration time required for the detection of faint emission lines in a newly proposed spectroscopic observation, and the detection of faint sources in a new imaging observation. We apply the results to the calculation of observation time required to claim the detection of the surface thermal emission from neutron stars with two virtual instruments.
\end{abstract}

Key words : methods: statistical - methods: data analysis - instrumentation: miscellaneous

\section{INTRODUCTION}

Considering a measurement of Gaussian signals in the presence of background that has been independently measured, the well-known "signal-to-noise" ratio $(S / N$ or $\mathrm{SNR})$, is usually estimated to assess the statistical significance of the background-subtracted signal (e.g., Huffman 1992; Bevington \& Robinson 2002). Gehrels (1986) and Ebeling (2003, 2004) investigated Poisson confidence limits for small numbers of events in astrophysical data, and derived approximate formulae for the confidence limits. Feldman \& Cousins (1998) clearly illustrated the discrepancies between the treatment of upper confidence limits for null results and two-sided confidence intervals for non-null results, commonly found in high energy physics literatures, and developed a confidence belt construction based on the "ordering principle" which unifies the treatment of upper confidence limits and of confidence intervals. Its improvements also have been proposed by several authors (Giunti 1999; Roe 1999). These investigations are related to the a posteriori claim whether a source signal was detected in an already performed experiment.

Now, suppose that we have a theory that predicts a certain amount of source signal, and from instruments we predict how much background will be observed. One would like to know whether the numbers of these expected events will allow, in advance, a particular experiment to claim a discovery at a certain statistical significance level. The "signal-to-noise" ratio is used widely as a measure of detection capability. However, the ob- served number of source-signal events may have only $50 \%$ chance (if the events were drawn from the symmetric probability distribution centered at their "true" mean value) to exceed the claimed significance level, as illustrated in Figure 1.

Here, we have to discriminate two concepts on the detection capabilities: one related to the a posteriori claim or decision whether a source signal was observed in the previously preformed experiment, and the other related to the a priori prediction of the detection limit in a newly proposed, but not yet performed, experiment. The "signal-to-noise" ratio and the works done by particle physicists (e.g., Feldman \& Cousins 1998) are, in fact, related with the a posteriori decision.

In his pioneering work, Currie (1968) clearly demonstrated the differences between two concepts of detectability, namely the a posteriori "critical" and a priori "detection" limits, which are firmly based on the statistical theory of hypothesis testing, and presented working formulae for the conventional assumption of a Gaussian signal distribution (see also, Currie 1972, 1995). More recently, Hernandez (1996) also emphasized that the "detectability" is not at all the same as "deciding" whether a real signal has been detected, given an observed signal, and proposed basically the same concepts as the "critical" and "detection" limits defined in Currie (1968, 1972, 1995). Bityukov \& Krasnikov $(1999,2000)$ also noted the difference between two concepts and derived a simple but useful formula for the detection limit by applying Gaussian 


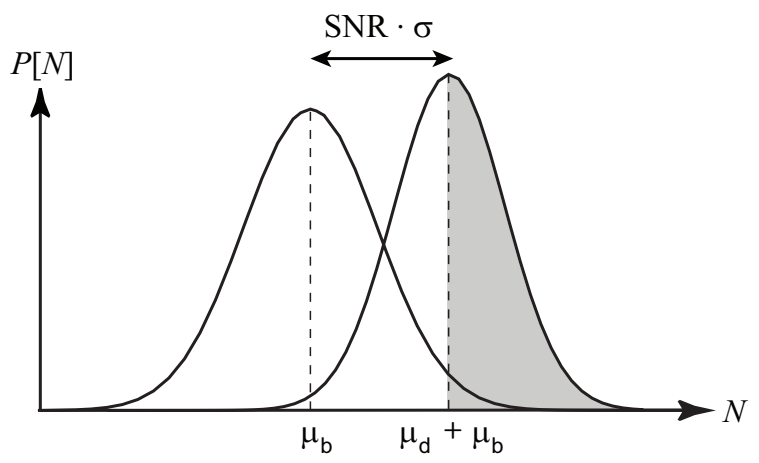

Fig. 1. - Conventional definition of "detection" limit $\left(\mu_{\mathrm{d}}\right)$ based on the "signal-to-noise" ratio (SNR), in which the detection limit is claimed to be the SNR (usually, $=3$ or 5 ) times the standard deviation $\sigma$. Here, $\mu_{\mathrm{b}}$ denotes the mean value of background. There will be only $50 \%$ chance (gray area) for the measured number of events to exceed the claimed significance level, SNR.

approximation to Poission signals.

However, astronomers still often use the "signal-tonoise" ratio, which is the statistical significance of an observed signal, for testing the possibility of detecting a desired source signal in a new proposal (e.g., EUVE GO Center 1997; Biretta \& Heyer 2001). The observed significance level would be lower than the expected one, or the signal may not be even observed at all, when its detection is claimed using the signal-to-noise ratio, mainly due to the statistical fluctuations of the source and background signals. In fact, the required integration time, when the signal-to-noise ratio is used, is underestimated, and the detection of the signal could not be guaranteed at the claimed confidence level. Correct assessment of the detection capability is crucial especially in an experiment to be proposed for the detection of a faint source.

In this paper, these two concepts defined in Currie (1968) are summarized, and the approximate equations evaluating the detection capabilities for Poisson signals are found, based on these definitions. We propose an algorithm, simple but still accurate, for the evaluation of the detection limits for a given significance level. We also apply the results to the prediction of observation time required to detect thermal emission from the surfaces of neutron stars with two virtual instruments, one

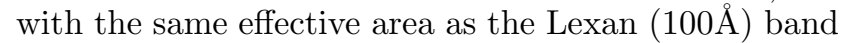
of the Extreme Ultraviolet Explorer (EUVE) scanning telescopes, and the other with 10 times higher effective area.

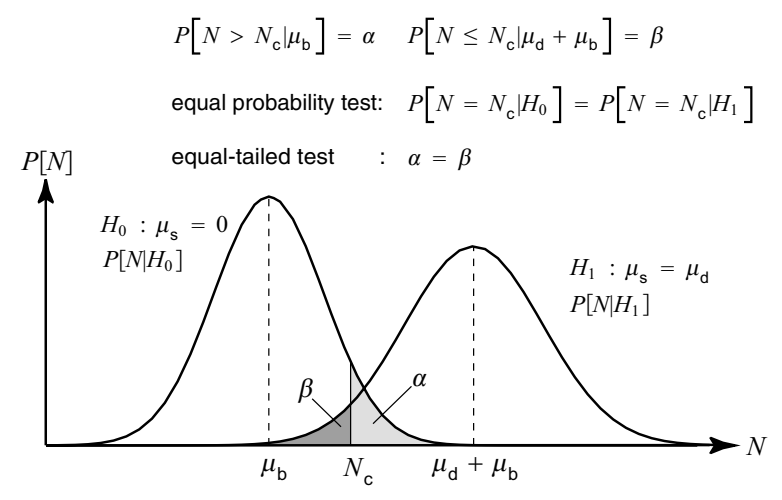

Fig. 2.- Definitions of "critical" (or decision) limit $\left(N_{\mathrm{c}}\right)$ and "detection" limit $\left(\mu_{\mathrm{d}}\right)$ based on hypothesis testing.

\section{DEFINITIONS OF CRITICAL AND DE- TECTION LIMITS}

Throughout this paper, the symbols $\mu, N$, and $\sigma$ will be used to denote the "true" mean, the "observed" or "random" values, and the standard deviation, respectively. The background and source signal values will be denoted using the subscripts 'b' and 's', respectively.

Based on the statistical theory of hypothesis testing, two limiting levels have been defined by Currie (1968, 1972, 1995): (1) the "critical" or "decision" limit $N_{\mathrm{c}}$, the signal level above which an observed signal may be reliably recognized as "detected" a source signal, and (2) the "detection" limit or "minimum detectable" limit $\mu_{\mathrm{d}}$, the "true" mean value of the source signal that may be expected a priori to lead to detection in a planned and specified experiment. The first aspect is thus related to the making of an a posteriori "decision" based upon the observation and a definite criterion for detection. The second aspect is related to the making of an a priori "prediction" of the detection capabilities of a given measurement process.

We use a classic one-sided test to define the minimum ("critical") threshold $N_{\mathrm{c}}$ that should be accepted as a real source signal with a certain probability $(1-\alpha)$, and then define the "detection" threshold as being the theoretical level that its measured signal wouldn't fall below $N_{\mathrm{c}}$ with another probability $(1-\beta)$. In hypothesis testing, decisions are subject to two kinds of error: deciding that the source signal is present when it is not (with probability $\alpha$; error of the first kind), and failing to decide that it is present when it is (with probability $\beta$; error of the second kind). The decision "detected" or "not detected" is made by comparison of the observed quantity $(N)$ with the "critical value" $\left(N_{\mathrm{c}}\right)$ of the relevant distribution, such that the probability of exceeding $N_{\mathrm{c}}$ is no greater than $\alpha$ if the true source signal is absent $\left(\mu_{\mathrm{s}}=0\right.$; null hypothesis $\left.\mathrm{H}_{0}\right)$. Thus, the probability distribution of possible events, when the 
true source signal is absent, intersects $N=N_{\mathrm{c}}$ such that a fraction, $1-\alpha$, corresponds to the (correct) decision, "not detected". The above definition of $N_{\mathrm{c}}$ can be expressed as:

$$
P\left[N>N_{\mathrm{c}} \mid \mu_{\mathrm{b}}\right] \leq \alpha,
$$

where $P[X \mid \mu]$ denotes a probability pertaining to a random variable $X$ with a true mean value $\mu$. Generally, the equation is stated as an equality, but the inequality is also given to accommodate discrete distributions, such as Poisson distributions, where not all values of $\alpha$ are possible. The $N_{c}$ is defined as the minimum value which satisfy the above equation, when the discrete distributions are concerned.

The "detection limit" $\mu_{\mathrm{d}}$ is defined as the true value of the source signal having a $1-\beta$ probability of being detected when the source signal is present $\left(\mu_{\mathrm{s}}=\mu_{\mathrm{d}}\right.$; alternative hypothesis $\mathrm{H}_{1}$ ), and with a maximum $\alpha$ probability of falsely interpreting the background event as source signal. The detection limit is thus the true value of the source signal for which the probability that the observed value $N$ does not exceed $N_{\text {c }}$ is $\beta$. The definition of $\mu_{\mathrm{d}}$ can be expressed as

$$
P\left[N \leq N_{\mathrm{c}} \mid \mu_{\mathrm{d}}+\mu_{\mathrm{b}}\right]=\beta .
$$

The relationship between the critical and detection limits is illustrated in Figure 2. It should be noted that the critical value $N_{\mathrm{c}}$ need not be defined either as the intersection point of two probability distribution curves, where the distribution functions have the same values (equal probability test), or as the point where the errors $(\alpha, \beta)$ are the same (equal-tailed test).

Since almost all the distributions we might encounter become normally distributed as the mean value gets large, it might be natural to define the statistical significance $z_{0}$ of an observed signal $N_{0}$ such that $P[N>$ $\left.N_{0} \mid \mu_{\mathrm{b}}\right] \equiv P\left[Z>z_{0}\right]$, where $Z$ denotes a random variable following the standard normal distribution with zero mean and unit variance, as defined in Gehrels (1986), Narsky (2000), and Ebeling (2003). The value $z_{0}$ is then the equivalent Gaussian number of $\sigma$ corresponding to the significance level, and is a function of $N_{0}$ and $\mu_{\mathrm{b}}$.

The definition of the statistical significance $z_{0}$ for a given signal $N_{0}$ may be represented as a functional form, $\widehat{S}_{\mathrm{c}}\left(N_{0}, \mu_{\mathrm{b}}\right)=z_{0}$. Inversely, the function $\widehat{S}_{\mathrm{c}}\left(N_{0}, \mu_{\mathrm{b}}\right)$ is now an estimator for the calculation of observed signal $N_{0}$ given a statistical significance $z_{0}$. Then, the critical limit $N_{\mathrm{c}}$ for a given error probability $\alpha$ of the first kind can be found using the formula, $\widehat{S}_{\mathrm{c}}\left(N_{\mathrm{c}}, \mu_{\mathrm{b}}\right)=$ $z_{1-\alpha}$, where $z_{1-\alpha}$ denotes the $(1-\alpha)$-quantile of the standard normal distribution. The $q$-quantile of a distribution, $x_{q}$, is defined by $P\left[x \leq x_{q}\right]=q(0 \leq q \leq 1)$. Similarly, the significance $z_{0}^{\prime}$ of the true mean value $\mu_{\mathrm{s}}=\mu_{0}$ may be defined by $P\left[N \leq N_{\mathrm{c}} \mid \mu_{0}+\mu_{\mathrm{b}}\right] \equiv$ $P\left[Z \geq z_{0}^{\prime}\right]$. Again, the definition of the significance $z_{0}^{\prime}$ can be represented by $\widehat{S}_{\mathrm{d}}\left(N_{\mathrm{c}}, \mu_{0}+\mu_{\mathrm{b}}\right)=z_{0}^{\prime}$. Then, the true mean detection limit $\mu_{\mathrm{d}}$, given a significance $z_{1-\beta}$, can be found by $\widehat{S}_{\mathrm{d}}\left(N_{\mathrm{c}}, \mu_{\mathrm{d}}+\mu_{\mathrm{b}}\right)=z_{1-\beta}$, and the function $\widehat{S}_{\mathrm{d}}$ is an estimator of the detection limit $\mu_{\mathrm{d}}$ for the given significance $z_{1-\beta}$.

The previous definitions of the critical and detection limits can be easily understood in the case of Gaussian signals. Given the significances $z_{1-\alpha}$ and $z_{1-\beta}$, the critical and detection limits are simply given by

$$
\begin{aligned}
N_{\mathrm{c}} & =\mu_{\mathrm{b}}+z_{1-\alpha} \sigma_{\mathrm{b}}, \quad \text { and } \\
\mu_{\mathrm{d}}+\mu_{\mathrm{b}} & =N_{\mathrm{c}}+z_{1-\beta} \sigma_{\mathrm{s}+\mathrm{b}},
\end{aligned}
$$

where $\sigma_{\mathrm{b}}$ and $\sigma_{\mathrm{s}+\mathrm{b}}$ denote the standard deviations of the background and total (source+background) signals, respectively. These result in

$$
\begin{aligned}
\mu_{\mathrm{d}} & =z_{1-\alpha} \sigma_{\mathrm{b}}+z_{1-\beta} \sigma_{\mathrm{s}+\mathrm{b}} \\
& =z_{1-\alpha} \sigma_{\mathrm{b}}+z_{1-\beta} \sqrt{\sigma_{\mathrm{s}}^{2}+\sigma_{\mathrm{b}}^{2}} .
\end{aligned}
$$

Here, $\sigma_{\mathrm{s}}$ is the standard deviation of the source signal. In the case of Gaussian approximation of Poisson signals, the equation becomes implicit in terms of $\mu_{\mathrm{d}}$. Its explicit solution and approximations are described in Appendix B.

\section{DETECTION LIMIT FOR POISSON SIG- NALS}

If a random variable $N$ follows a Poisson distribution with a true mean $\mu$, then for any non-negative integer $n$, the probability that $N=n$ is given by

$$
P[N=n \mid \mu]=\frac{e^{-\mu} \mu^{n}}{n !} .
$$

There may be no value $N_{\mathrm{c}}$, for a given $\mu$, that satisfies the equality in equation (1) exactly, because the observed or random variable $N$ assumes only discrete values. The critical value in this case is defined as the smallest value $N_{\mathrm{c}}$ such that $P\left[N>N_{\mathrm{c}} \mid \mu_{\mathrm{b}}\right] \leq \alpha$, and thus

$$
P\left[N>N_{\mathrm{c}} \mid \mu_{\mathrm{b}}\right] \leq \alpha<P\left[N>N_{\mathrm{c}}-1 \mid \mu_{\mathrm{b}}\right] .
$$

The exact results for Poisson distributions are then easily calculated with incomplete gamma functions. The Poisson distribution is also related to a chi-square distribution by the formulae,

$$
\begin{aligned}
P[N \leq n \mid \mu] & =P\left[\chi^{2}(2 n+2) \geq 2 \mu\right], \text { and } \\
P[N>n \mid \mu] & =1-P[N \leq n \mid \mu] \\
& =P\left[\chi^{2}(2 n+2)<2 \mu\right],
\end{aligned}
$$

where $\chi^{2}(\nu)$ denotes a chi-square random variable with $\nu$ degrees of freedom (Abramowitz \& Stegun 1972). Using these relationships between the Poisson and chisquare distributions, the critical and detection limits for the Poisson signal can be obtained from:

$$
\begin{gathered}
P\left[\chi^{2}\left(2 N_{\mathrm{c}}+2\right)<2 \mu_{\mathrm{b}}\right] \leq \alpha<P\left[\chi^{2}\left(2 N_{\mathrm{c}}\right)<2 \mu_{\mathrm{b}}\right], \\
\beta=P\left[\chi^{2}\left(2 N_{\mathrm{c}}+2\right) \geq 2\left(\mu_{\mathrm{d}}+\mu_{\mathrm{b}}\right)\right] .
\end{gathered}
$$


TABLE 1.

RITICAL AND DETECTION LIMITS FOR POISSON SIGNALS WHEN $\alpha=\beta=0.05$.

\begin{tabular}{|c|c|c|}
\hline$\mu_{\mathrm{b}}$ & $\overline{\bar{N}}$ & $\mu_{\mathrm{d}}+\mu_{\mathrm{b}}$ \\
\hline $0.000-$ & 0 & 2.996 \\
\hline $0.051-$ & 1 & 4.744 \\
\hline $0.355-$ & 2 & 6.296 \\
\hline $0.818-$ & 3 & 7.754 \\
\hline $1.366-$ & 4 & 9.154 \\
\hline $1.970-$ & 5 & 10.513 \\
\hline $2.613-$ & 6 & 11.842 \\
\hline $3.285-$ & 7 & 13.148 \\
\hline $3.981-$ & 8 & 14.435 \\
\hline $4.695-$ & 9 & 15.705 \\
\hline $5.425-$ & 10 & 16.962 \\
\hline $6.169-$ & 11 & 18.208 \\
\hline $6.924-$ & 12 & 19.443 \\
\hline $7.690-8.464$ & 13 & 20.669 \\
\hline $8.464-9.246$ & 14 & 21.887 \\
\hline $9.246-10.036$ & 15 & 23.097 \\
\hline $10.036-10.832$ & 16 & 24.301 \\
\hline $10.832-11.634$ & 17 & 25.499 \\
\hline $11.634-12.442$ & 18 & 26.692 \\
\hline $12.442-13.255$ & 19 & 27.879 \\
\hline $13.255-14.072$ & 20 & 29.062 \\
\hline $14.072-14.894$ & 21 & 30.240 \\
\hline $14.894-15.720$ & 22 & 31.415 \\
\hline $15.720-16.549$ & 23 & 32.585 \\
\hline $16.549-17.382$ & 24 & 33.752 \\
\hline $17.382-18.219$ & 25 & 34.916 \\
\hline $18.219-19.058$ & 26 & 36.077 \\
\hline $19.058-19.901$ & 27 & 37.234 \\
\hline $19.901-20.746$ & 28 & 38.389 \\
\hline $20.746-21.594$ & 29 & 39.541 \\
\hline
\end{tabular}

It is clear from equation (9) that the true mean values of background signals in the range,

$$
\frac{1}{2} \chi_{\alpha}^{2}\left(2 N_{\mathrm{c}}\right)<\mu_{\mathrm{b}} \leq \frac{1}{2} \chi_{\alpha}^{2}\left(2 N_{\mathrm{c}}+2\right) \quad\left(N_{\mathrm{c}}=0,1,2, \ldots\right),
$$

yield the same critical limit $N_{\mathrm{c}}$. Here, $\chi_{q}^{2}(\nu)$ denotes the $q$-quantile of the chi-square distribution. The detection limit $\mu_{\mathrm{d}}$ of the source signal is then found using the following formula:

$$
\mu_{\mathrm{d}}+\mu_{\mathrm{b}}=\frac{1}{2} \chi_{1-\beta}^{2}\left(2 N_{\mathrm{c}}+2\right) .
$$

Now, we can tabulate the range of background values $\mu_{\mathrm{b}}$ and the detection limits for given $N_{\mathrm{c}}, \alpha$, and $\beta$ values, using the equations (11) and (12). The numerical solutions, provided to 3 decimal places, are given in Table 1 for the case $\alpha=\beta=0.05$. In Figure 3 are shown the exact solution ranges, plotted as the discontinuous lines. The breaks in the table and the figure occur at $\mu_{\mathrm{b}}=\frac{1}{2} \chi_{\alpha}^{2}\left(2 N_{\mathrm{c}}\right)$ and $\frac{1}{2} \chi_{\alpha}^{2}\left(2 N_{\mathrm{c}}+2\right)$.

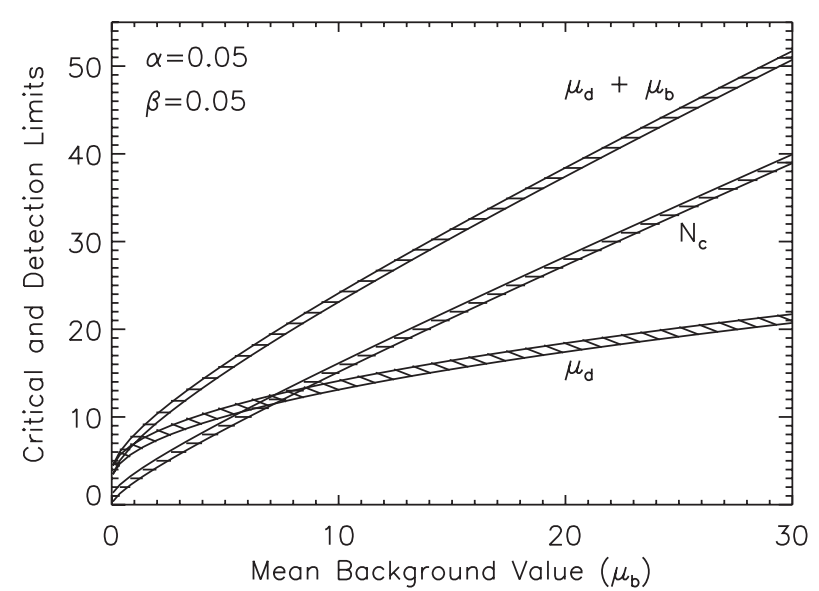

Fig. 3. - Critical and detection limits for Poisson signals when $\alpha=\beta=0.05$. The exact solution ranges are shown as discontinuous lines, and the approximate solutions that enclose the exact solutions as continuous lines.

The figure also shows two approximate solutions, found in Appendix A, as the continuous lines. In the appendix, we obtained two approximate estimators for the critical limit, $\widehat{S}_{\mathrm{c}}^{\text {up }}$ and $\widehat{S}_{\mathrm{c}}^{\text {low }}$, corresponding to the upper and lower bounds in equation (9). By substituting these approximations, two approximate estimators for the detection limit, $\widehat{S}_{\mathrm{d}}^{\text {up }}$ and $\widehat{S}_{\mathrm{d}}^{\text {low }}$, were also found. The approximations in the figure were then obtained using these approximate estimators. We also proposed an approximate estimator, $\widehat{S}^{*} \equiv \sqrt{\mu_{\mathrm{d}}+\mu_{\mathrm{b}}}-\sqrt{\mu_{\mathrm{b}}}$, to estimate easily the detection limit in the equal-tailed test $(\alpha=\beta)$.

\section{APPLICATION: THERMAL EMISSION FROM NEUTRON STARS' SURFACES}

The integration time or observation time, which is often required in observational proposals to justify the detection of faint signals, can be estimated using the estimator $\widehat{S}^{*}$, suggested in Appendix A, if the source and background fluxes are given in units of counts per unit time. The integration time $T$, required to claim the detection at a centain confidence level $z_{1-\alpha}$, is then given by $T=z_{1-\alpha}^{2} /\left(\sqrt{F_{\mathrm{s}}+F_{\mathrm{b}}}-\sqrt{F_{\mathrm{b}}}\right)^{2}$, for a given source flux $F_{\mathrm{s}}$ and background flux $F_{\mathrm{b}}$.

As an example, we calculate the observation time to detect the surface thermal emission from neutron stars, assuming an instrument with the same effective area as the EUVE mission. Seon \& Edelstein (1998) and Korpela \& Bowyer (1998) reported the results of searches for EUV emission from neutron stars conducted with the EUVE scanning telescopes. They derived limits to the temperature of surface thermal radiation from the objects. Old neutron stars are expected to emit significant EUV only with the presence of some form of 

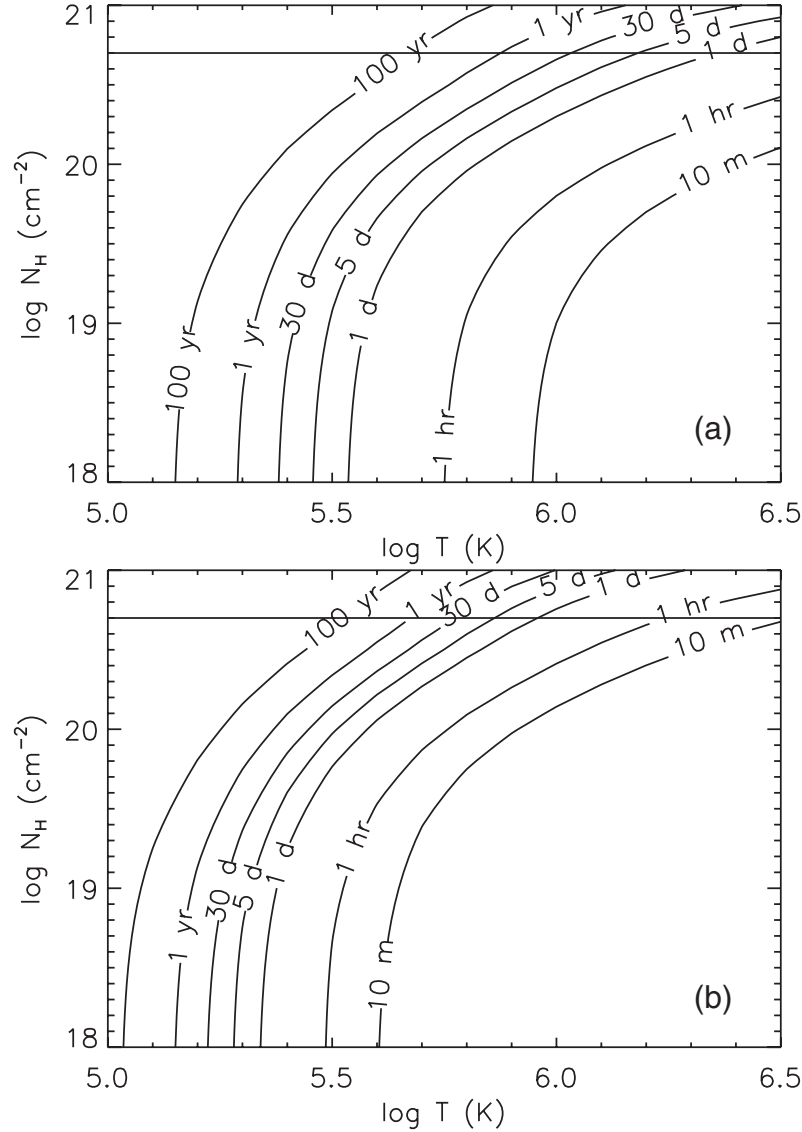

Fig. 4.- Observation time required to claim the detection of surface thermal radiation from neutron stars at the confidence level of $3 \sigma\left(z_{1-\alpha}=z_{1-\beta}=3\right)$. The calculation was performed assuming (a) an instrument with the same effec-

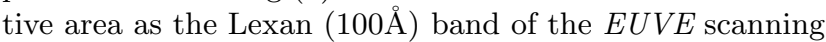
telescopes, and (b) an instrument with 10 times larger effective area than the Laxan band. The horizontal lines represent a typical hydrogen column density of $N_{\mathrm{HI}}=5 \times 10^{20}$ $\mathrm{cm}^{-2}$. The symbols ' $\mathrm{m}$ ', ' $\mathrm{hr}$ ', 'd', and 'yr' represent minutes, hours, days, and years, respectively.

reheating mechanism Becker \& Trümper 1993). It may be, thus, valuable, not only as an example of the detection limit calculation but also as a reference for future EUV missions, to demonstrate the calculation of observation time required for the detection of the surface thermal radiation with the $E U V E$ scanning telescopes.

We estimated the observation time required to claim the detection of surface thermal emission from neutron stars, at the confidence level of $3 \sigma\left(z_{1-\alpha}=z_{1-\beta}=3\right)$, for various blackbody temperatures $T$ and absorbing hydrogen column densities $N_{\mathrm{HI}}$. Figure 4 shows contours of the observation times using the source radius of $10 \mathrm{~km}$ at an object distance of $1 \mathrm{~km}$, the background count rate of 0.0074 counts/s, averaged over the values obtained in Seon \& Edelstein (1998), and the effective area of the Lexan $(100 \AA)$ band of the EUVE scanning telescope (Bowyer et al. 1996). The figure also shows the contours of the observation times for an instrument with 10 times larger effective area than the EUVE scanning telescope. It is found that the emission with temperatures of $\lesssim 1.0 \times 10^{6} \mathrm{~K}$ (for the first instrument) and $\lesssim 5.0 \times 10^{5} \mathrm{~K}$ (for the second instrument), absorbed by interstellar medium with a hydrogen column density of $N_{\mathrm{HI}}=5^{20} \mathrm{~cm}^{-2}$, can not be detected during the feasible observation time of $<30$ days. The results are consistent with the non-detection of the surface thermal emission of old neutron stars analyzed by Seon \& Edelstein (1998) and Korpela \& Bowyer (1998).

\section{SUMMARY}

We derived the formulae for the calculation of the "detection limit" of Poisson events, following its correct definition. Applying an equal-tailed test, in which the false-identification error probabilities $\alpha$ and $\beta$ are the same, the formula $\sqrt{\mu_{\mathrm{d}}+\mu_{\mathrm{b}}}-\sqrt{\mu_{\mathrm{b}}}=z_{1-\beta}$ is found to be a reliable estimator of the detection limit for Poisson events. An algorithm, simple yet accurate, was also proposed in Appendix A to estimate $N_{\mathrm{c}}$ and $\mu_{\mathrm{d}}$, which used the approximate equations for the critical limit, and the $q$-quantile of the chi-square distribution. It is found that this algorithm gives fairly accurate solutions for the detection limit $\mu_{\mathrm{d}}$ for any $\alpha$ and $\beta$.

\section{REFERENCES}

Abramowitz, M. \& Stegun, I. A., 1972, Handbook of Mathematical Functions (9th ed.; New York: Dover)

Becker, W. \& Trümper, J., 1993, Detection of Pulsed X-Rays from the Binary Millisecond Pulsar J:04374715, Nature, 365, 528

Bevington, P. \& Robinson, D. K., 2002, Data Reduction and Error Analysis for the Physical Sciences (3rd ed.; New York: McGraw-Hill)

Biretta, J. \& Heyer, I., 2001, WFPC2 Instrument Handbook, Version 6.0 (Baltimore: STScI)

Bityukov, S. I. \& Krasnikov, N. V., 1998, New Physics Discovery Potential in Future Experiments, Mod. Phys. Lett., A13, 3235

Bityukov, S. I. \& Krasnikov, N. V., 2000, On the observability of a signal above background, Nucl. Instrum. Methods A, 452, 518

Bowyer, S., Lampton, M., Lewis, J., Wu, X., Jelinsky, P., \& Malina, R. F., 1996, The Second ExtremeUltraviolet Explorer Source Catalog, ApJS, 102, 129

Currie, L. A., 1968, Limits for Qualitative Detection and Quantitative Determination , Analyt. Chem., 40,586

Currie, L. A., 1972, The Measurement of Environmental Levels of Rare Gas Nuclides and the Treatment of Very Low-Level Counting Data, IEEE Trans. Nucl. Sci., NS19 (1), 119 
Currie, L. A., 1995, Nomenclature in Evaluation of Analytical Methods including Detection and Quantification Capabilities, Pure \& Appl. Chem., 67, 1699

Ebeling, H., 2003, Improved approximations of Poissonian errors for high confidence levels, MNRAS, 340, 1269

Ebeling, H., 2004, Erratum: Improved approximations of Poissonian errors for high confidence levels, MNRAS, 349, 768

EUVE Guest Observer Center 1997, EUVE Guest Observer Program Handbook, UVB/EUVE Call for Observing Proposals 97-EUVE-06, Appendix C (Berkeley: Center for EUV Astrophysics)

Feldman, G. J. \& Cousins, R. D., 1998, Unified approach to the classical statistical analysis of small signals, Phys. Rev. D, 57, 003873

Gehrels, N., 1986, Confidence limits for small numbers of events in astrophysical data, ApJ, 303, 336

Giunti, C., 1999, A new ordering principle for the classical statistical analysis of Poisson processes with background, Phys. Rev. D, 59, 053001

Hernandez, J. J., Navas, S., \& Rebecchi, P., 1996, Estimating exclusion limits in prospective studies of searches, Nucl. Instr. Methods, A378, 301

Huffman, R. E., 1992, Atmospheric Ultraviolet Remote Sensing (Boston: Academic Press, Inc.), 32

Korpela, E. J. \& Bowyer, S., 1998, Extreme Ultraviolet Explorer Observations of Neutron Stars, AJ, 115, 2551

Narsky, I., 2000, Estimation of upper limits using a Poisson statistic, Nucl. Instr. Methods, A450, 444

Roe, B. P., 1999, Improved Probability Method for Estimating Signal in the Presence of Background, Phys. Rev. D, 60, 053009

Seon, K.-I. \& Edelstein, J., 1998, Extreme-Ultraviolet Observations of Nine Pulsars, AJ, 115, 2097

\section{APPENDIX A. Approximations for Poisson sig- nals}

The random variable $\sqrt{2 \chi^{2}(\nu)}$ becomes normally distributed, as $\nu \rightarrow \infty$, with a mean $Z_{0}(\nu) \equiv \sqrt{2 \nu-1}\{1+$ $\left.O\left(\nu^{-2}\right)\right\}$, and a variance $\sigma^{2}(\nu) \equiv 1+O\left(\nu^{-1}\right)$ (Abramowitz $\&$ Stegun 1972). The cumulative chi-square distribution can thus be approximated as a cumulative normal distribution, as follows:

$$
P\left[\chi^{2}(\nu)<\chi_{0}^{2}\right] \approx P\left[Z<\frac{\sqrt{2 \chi_{0}^{2}}-Z_{0}(\nu)}{\sigma(\nu)}\right],
$$

where $Z$ denotes a random variable following the standard normal distribution. Using this relation, the definition of estimator, and the fact that $P[Z<-z]=$
$P[Z>z]$ for positive $z$, equation (9) can be rewritten with two estimators for the critical limit,

$$
\widehat{S}_{\mathrm{c}}^{\text {low }}<z_{1-\alpha} \leq \widehat{S}_{\mathrm{c}}^{\text {up }} .
$$

Here, two estimators are then approximately given by

$$
\begin{aligned}
& \widehat{S}_{\mathrm{c}}^{\text {up }} \approx \frac{Z_{0}\left(2 N_{\mathrm{c}}+2\right)-\sqrt{4 \mu_{\mathrm{b}}}}{\sigma\left(2 N_{\mathrm{c}}+2\right)}, \text { and } \\
& \widehat{S}_{\mathrm{c}}^{\text {low }} \approx \frac{Z_{0}\left(2 N_{\mathrm{c}}\right)-\sqrt{4 \mu_{\mathrm{b}}}}{\sigma\left(2 N_{\mathrm{c}}\right)}
\end{aligned}
$$

Solving equation (A3) for $N_{\mathrm{c}}$, a range enclosing the integral critical limit for a given significance $z_{1-\alpha}$ can be found using the equation,

$$
N_{\mathrm{c}}^{\text {low }} \leq N_{\mathrm{c}}<N_{\mathrm{c}}^{\mathrm{up}}
$$

where the upper and lower bounds are approximately given by

$$
\begin{aligned}
N_{\mathrm{c}}^{\mathrm{up}} \approx & \mu_{\mathrm{b}}+z_{1-\alpha} \sqrt{\mu_{\mathrm{b}}}+\frac{z_{1-\alpha}^{2}+1}{4} \\
& +O\left(\mu_{\mathrm{b}}^{-1 / 2}\right), \text { and } \\
N_{\mathrm{c}}^{\text {low }}= & N_{\mathrm{c}}^{\mathrm{up}}-1 .
\end{aligned}
$$

It should be noted that the $N_{\mathrm{c}}^{\text {low }}$ and $N_{\mathrm{c}}^{\text {up }}$ values, corresponding to $\widehat{S}_{\mathrm{c}}^{\text {up }}$ and $\widehat{S}_{\mathrm{c}}^{\text {low }}$, respectively, are not necessarily integers, but real numbers. The curves corresponding to $N_{\mathrm{c}}^{\text {low }}$ and $N_{\mathrm{c}}^{\text {up }}$ are shown in Figure 3 . The real upper bound $N_{\mathrm{c}}^{\text {up }}$ coincides approximately with the integer $N_{\mathrm{c}}=\left[N_{\mathrm{c}}^{\mathrm{up}}\right]$ at the mean background value given by $\mu_{\mathrm{b}}=(1 / 2) \chi_{\alpha}^{2}\left(2 N_{\mathrm{c}}\right)$, and the real value $N_{\mathrm{c}}^{\text {low }}$ with integer $N_{\mathrm{c}}-1$ at $\mu_{\mathrm{b}}=(1 / 2) \chi_{\alpha}^{2}\left(2 N_{\mathrm{c}}+2\right)$. Here, the bracket [ ] denotes the largest integer smaller than or equal to the specified value. It is now clear that these lower and upper bounds may be considered as the approximate boundaries enclosing the possible critical values. Thus, the integer critical value $N_{\mathrm{c}}$ is then given by $\left[N_{\mathrm{c}}^{\text {up }}\right]$ or $\left[N_{\mathrm{c}}^{\mathrm{up}}\right]-1$. Note that the approximate bounds $N_{\mathrm{c}}^{\text {low }}$ and $N_{\mathrm{c}}^{\text {up }}$ are slightly higher than the exact boundaries.

Using Eqs. (10) and (A1), the estimator of detection limit $\mu_{\mathrm{d}}$ can be expressed as follows:

$$
\widehat{S}_{\mathrm{d}} \approx \frac{\sqrt{4\left(\mu_{\mathrm{d}}+\mu_{\mathrm{b}}\right)}-Z_{0}\left(2 N_{\mathrm{c}}+2\right)}{\sigma\left(2 N_{\mathrm{c}}+2\right)} .
$$

By substituting two approximations of the critical value $N_{\text {c }}$ into the above equation, in order to express the estimator in terms of $\mu_{\mathrm{b}}$ and $\mu_{\mathrm{d}}$, two approximations for the estimator are found as follows:

$$
\begin{aligned}
& \widehat{S}_{\mathrm{d}}^{\text {low }} \approx 2\left(\sqrt{\mu_{\mathrm{d}}+\mu_{\mathrm{b}}}-\sqrt{\mu_{\mathrm{b}}}\right)-z_{1-\alpha}-1 / \sqrt{\mu_{\mathrm{b}}} \\
&+O\left(\mu_{\mathrm{b}}^{-1}\right), \\
& \widehat{S}_{\mathrm{d}}^{\mathrm{up}} \approx 2\left(\sqrt{\mu_{\mathrm{d}}+\mu_{\mathrm{b}}}-\sqrt{\mu_{\mathrm{b}}}\right)-z_{1-\alpha}+O\left(\mu_{\mathrm{b}}^{-1}\right), \quad \text { and } \\
& \widehat{S}_{\mathrm{d}}^{\text {low }}<\widehat{S}_{\mathrm{d}} \leq \widehat{S}_{\mathrm{d}}^{\text {up }} .
\end{aligned}
$$




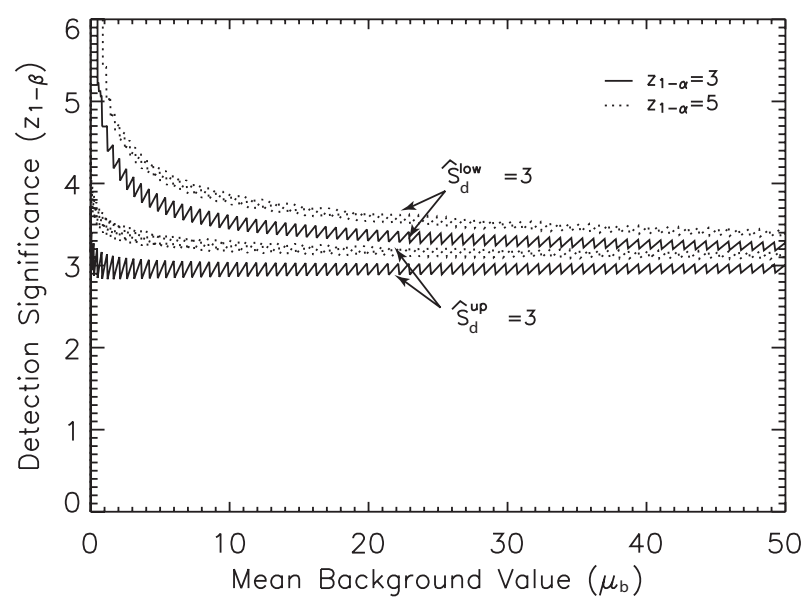

Fig. A1. - Dependences on the mean background value $\mu_{\mathrm{b}}$ of the true detection significances $z_{1-\beta}$, when the approximate estimators $\widehat{S}_{\mathrm{d}}^{\text {low }}$ and $\widehat{S}_{\mathrm{d}}^{\text {up }}$ are used to calculated the detection limits.

Here, $\widehat{S}_{\mathrm{d}}^{\text {low }}$ and $\widehat{S}_{\mathrm{d}}^{\text {up }}$ correspond to $N_{\mathrm{c}}^{\mathrm{up}}$ and $N_{\mathrm{c}}^{\text {low }}$, respectively. The detection limit for a given significance $z_{1-\beta}$ is then given by two approximations:

$$
\begin{aligned}
\mu_{\mathrm{d}}^{\text {low }} \approx & \frac{\left(z_{1-\alpha}+z_{1-\beta}\right)^{2}}{4}+\left(z_{1-\alpha}+z_{1-\beta}\right) \sqrt{\mu_{\mathrm{b}}} \\
& +O\left(\mu_{\mathrm{b}}^{-1 / 2}\right) \\
\mu_{\mathrm{d}}^{\text {up }} \approx & \mu_{\mathrm{d}}^{\text {low }}+1, \text { and } \\
\mu_{\mathrm{d}}^{\text {low }} \leq & \mu_{\mathrm{d}}<\mu_{\mathrm{d}}^{\text {up }} .
\end{aligned}
$$

Again, $\mu_{\mathrm{d}}^{\text {up }}$ and $\mu_{\mathrm{d}}^{\text {low }}$ are derived from $\widehat{S}_{\mathrm{d}}^{\text {low }}$ and $\widehat{S}_{\mathrm{d}}^{\text {up }}$, respectively. Note that, for the equal-tailed test $(\alpha=$ $\beta)$, the approximation $\mu_{\mathrm{d}}^{\text {low }}$ approaches the detection limit obtained from the Gaussian approximation shown in equation (B8).

Figure A1 shows the dependences of the true detection significances on the mean background value $\mu_{\mathrm{b}}$, when the detection limits are calculated using the approximate limits $\mu_{\mathrm{d}}^{\text {low }}$ and $\mu_{\mathrm{d}}^{\text {up }}$. Here, the upper two curves are found using $\widehat{S}_{\mathrm{d}}^{\text {low }}=3$, and the lower two curves using $\widehat{S}_{\mathrm{d}}^{\mathrm{up}}=3$. The lowest curve, corresponding to $\alpha=\beta$, shows good agreement with the true significance values, $z_{1-\beta}=3$, as illustrated in Figure A1. In this equal-tailed test, both approximations $\widehat{S}_{\mathrm{d}}^{\text {low }}=z_{1-\alpha}$ and $\widehat{S}_{\mathrm{d}}^{\text {up }}=z_{1-\alpha}$ in Eq. (A7) result in $\sqrt{\mu_{\mathrm{d}}+\mu_{\mathrm{b}}}-\sqrt{\mu_{\mathrm{b}}}=z_{1-\beta}$ ignoring the $O\left(\mu_{\mathrm{b}}^{-1 / 2}\right)$ and lower orders. Thus, the formula $\widehat{S}^{*} \equiv \sqrt{\mu_{\mathrm{s}}+\mu_{\mathrm{b}}}-\sqrt{\mu_{\mathrm{b}}}$ is suggested as a reliable estimator of the detection limit $\mu_{\mathrm{d}}$, when $\alpha=\beta$ (equal-tailed test). However, the estimator $\widehat{S}^{*}$ yields relatively large errors when $\alpha \neq \beta$, when compared to the equal-tailed test, as can be seen in Figure A1.
It can be time-consuming to construct a list like Table 1 , where a critical value $N_{\mathrm{c}}$ and a detection limit $\mu_{\mathrm{d}}$ are found, corresponding to a given $\mu_{\mathrm{b}}$. Thus, it is desirable to construct a simple but accurate algorithm for estimating the critical and detection limits, which is applicable even for $\alpha \neq \beta$. For large values of $\nu$, the quantiles $\chi_{q}^{2}(\nu)$ may be approximated using the following formula (Abramowitz \& Stegun 1972):

$$
\chi_{q}^{2}(\nu) \approx \nu\left(1-\frac{2}{9 \nu}+z_{q} \sqrt{\frac{2}{9 \nu}}\right)^{3} .
$$

This formula is useful for estimating the detection limit for a Poisson signal. Using this approximation and equation (11), an algorithm for estimating the critical and detection limits is proposed, as follows:

(a) Compute the critical value $N_{\mathrm{c}}^{\mathrm{up}}$ from equation (A5).

(b) Set $N_{\mathrm{c}}=\left[N_{\mathrm{c}}^{\mathrm{up}}\right]-1$ if $\mu_{\mathrm{b}} \leq 0.5 \chi_{\alpha}^{2}\left(2\left[N_{\mathrm{c}}^{\mathrm{up}}\right]\right)$, or set $N_{\mathrm{c}}=\left[N_{\mathrm{c}}^{\mathrm{up}}\right]$ if $\mu_{\mathrm{b}}>0.5 \chi_{\alpha}^{2}\left(2\left[N_{\mathrm{c}}^{\mathrm{up}}\right]\right)$. Here, the $\alpha$-quantile of the chi-square distribution is calculated using equation (A9), and the symbol [ ] denotes the largest integer smaller than or equal to the specified value.

(c) Compute $\mu_{\mathrm{d}}^{\text {app }}=\frac{1}{2} \chi_{1-\beta}^{2}\left(2 N_{\mathrm{c}}+2\right)-\mu_{\mathrm{b}}$ using equation (A9).

Table A1 shows the critical values, the lower and upper bounds of the detection limits, and their approximate solutions $\mu_{\mathrm{d}}^{\text {app }}$ obtained using the algorithm above. The errors of the detection limits $\mu_{\mathrm{d}}^{\text {app }}$ and of the true significances $z_{1-\beta}$ are also shown in the last two columns. The algorithm gives accurate values for the detection limits and their true significances within $4.5 \%$, even for worst cases listed in Table A1. In fact, the critical values $N_{\mathrm{c}}$ obtained using this algorithm are not approximate, and more accurate detection limits can be also estimated using more accurate formula for $\chi_{q}^{2}$.

\section{APPENDIX B. Approximations in the limit of large mean values}

In the limit of large mean values $(\mu \gg 1)$, a Poisson distribution approaches a normal distribution with $\sigma=\sqrt{\mu}$. If the distribution of the signal $N_{\mathrm{s}+\mathrm{b}}\left(=N_{\mathrm{b}}\right)$ under $\mathrm{H}_{0}$ is approximately normal with a well-known standard deviation $\sigma_{\mathrm{s}+\mathrm{b}}\left(=\sigma_{\mathrm{b}}\right)$, the critical value $N_{\mathrm{c}}$ and its estimator $\widehat{S}_{\mathrm{c}}$ are then given by, respectively,

$N_{\mathrm{c}}=\mu_{\mathrm{b}}+z_{1-\alpha} \sqrt{\mu_{\mathrm{b}}}$, and $\widehat{S}_{\mathrm{c}}=\left(N_{\mathrm{c}}-\mu_{\mathrm{b}}\right) / \sqrt{\mu_{\mathrm{b}}} \cdot(\mathrm{B} 1)$

The minimum detectable source signal $\mu_{\mathrm{s}}=\mu_{\mathrm{d}}$ is determined implicitly by the following equation:

$$
\mu_{\mathrm{d}}+\mu_{\mathrm{b}}=N_{\mathrm{c}}+z_{1-\beta} \sqrt{\mu_{\mathrm{d}}+\mu_{\mathrm{b}}} .
$$


TABLE A1

Approximate solutions of THE CRITICAL AND DETECTION LIMITS, AND THEIR ASSOCIATED ERRORS.

\begin{tabular}{|c|c|c|c|c|c|c|c|c|c|}
\hline & $\mu_{\mathrm{b}}$ & {$\left[N_{\mathrm{c}}^{\mathrm{up}}\right]$} & $N_{\mathrm{c}}$ & $\mu_{\mathrm{d}}^{\text {low }}$ & $\mu_{\mathrm{d}}^{\mathrm{up}}$ & $\mu_{\mathrm{d}}^{\mathrm{app}}$ & $\mu_{\mathrm{d}}$ & $\Delta \mu_{\mathrm{d}}(\%)$ & $\Delta z_{1-\beta}(\%)$ \\
\hline \multirow{7}{*}{ (a) } & 0.0 & 0 & 0 & 1.642 & - & 2.280 & 2.303 & -1.003 & -1.030 \\
\hline & 0.6 & 2 & 2 & 3.628 & 6.699 & 4.709 & 4.722 & -0.274 & -0.398 \\
\hline & 3.0 & 5 & 5 & 6.082 & 7.905 & 6.266 & 6.275 & -0.140 & -0.209 \\
\hline & 10.2 & 14 & 14 & 9.828 & 11.254 & 9.922 & 9.928 & -0.052 & -0.086 \\
\hline & 12.0 & 17 & 17 & 10.521 & 11.912 & 11.601 & 11.606 & -0.040 & -0.072 \\
\hline & 20.1 & 26 & 26 & 13.134 & 14.432 & 13.733 & 13.736 & -0.027 & -0.048 \\
\hline & 29.7 & 37 & 37 & 15.611 & 16.854 & 16.380 & 16.383 & -0.019 & -0.034 \\
\hline \multirow{7}{*}{ (b) } & 0.0 & 0 & 0 & 2.141 & - & 2.968 & 2.996 & -0.911 & -0.807 \\
\hline & 0.6 & 2 & 2 & 4.408 & 7.713 & 5.684 & 5.696 & -0.202 & -0.248 \\
\hline & 3.0 & 5 & 5 & 7.210 & 9.138 & 7.506 & 7.513 & -0.088 & -0.113 \\
\hline & 10.2 & 14 & 14 & 11.487 & 12.970 & 11.683 & 11.687 & -0.027 & -0.039 \\
\hline & 12.0 & 17 & 17 & 12.278 & 13.722 & 13.497 & 13.499 & -0.020 & -0.031 \\
\hline & 20.1 & 26 & 26 & 15.261 & 16.600 & 15.975 & 15.977 & -0.013 & -0.019 \\
\hline & 29.7 & 37 & 37 & 18.089 & 19.366 & 18.974 & 18.976 & -0.008 & -0.013 \\
\hline \multirow{7}{*}{ (c) } & 0.0 & 0 & 0 & 2.706 & - & 2.968 & 2.996 & -0.911 & -0.807 \\
\hline & 0.6 & 2 & 2 & 5.254 & 8.794 & 5.684 & 5.696 & -0.202 & -0.249 \\
\hline & 3.0 & 6 & 6 & 8.403 & 10.437 & 8.837 & 8.842 & -0.066 & -0.095 \\
\hline & 10.2 & 16 & 16 & 13.212 & 14.752 & 14.098 & 14.101 & -0.020 & -0.033 \\
\hline & 12.0 & 18 & 18 & 14.101 & 15.597 & 14.689 & 14.692 & -0.018 & -0.029 \\
\hline & 20.1 & 28 & 28 & 17.454 & 18.834 & 18.287 & 18.289 & -0.010 & -0.018 \\
\hline & 29.7 & 39 & 39 & 20.634 & 21.944 & 21.238 & 21.240 & -0.007 & -0.012 \\
\hline \multirow{7}{*}{ (d) } & 0.0 & 2 & 2 & 9.000 & - & 10.964 & 10.870 & 0.863 & 0.792 \\
\hline & 0.6 & 5 & 4 & 13.648 & 18.937 & 13.868 & 13.793 & 0.547 & 0.569 \\
\hline & 3.0 & 10 & 9 & 19.392 & 22.208 & 19.230 & 19.176 & 0.284 & 0.343 \\
\hline & 10.2 & 22 & 21 & 28.163 & 30.126 & 28.596 & 28.559 & 0.129 & 0.181 \\
\hline & 12.0 & 24 & 24 & 29.785 & 31.672 & 30.722 & 30.687 & 0.113 & 0.163 \\
\hline & 20.1 & 36 & 35 & 35.900 & 37.581 & 36.614 & 36.585 & 0.079 & 0.119 \\
\hline & 29.7 & 48 & 47 & 41.699 & 43.258 & 41.793 & 41.768 & 0.060 & 0.092 \\
\hline \multirow{7}{*}{ (e) } & 0.0 & 2 & 2 & 16.000 & - & 21.489 & 20.584 & 4.396 & 4.402 \\
\hline & 0.6 & 5 & 4 & 22.197 & 28.777 & 25.067 & 24.394 & 2.756 & 2.494 \\
\hline & 3.0 & 10 & 9 & 29.856 & 33.249 & 31.930 & 31.484 & 1.417 & 2.494 \\
\hline & 10.2 & 22 & 21 & 41.550 & 43.827 & 44.072 & 43.792 & 0.640 & 1.218 \\
\hline & 12.0 & 24 & 24 & 43.713 & 45.888 & 46.782 & 46.522 & 0.559 & 1.218 \\
\hline & 20.1 & 36 & 35 & 51.866 & 53.771 & 54.587 & 54.377 & 0.387 & 1.218 \\
\hline & 29.7 & 48 & 47 & 59.598 & 61.341 & 61.567 & 61.388 & 0.290 & 1.218 \\
\hline \multirow{7}{*}{ (f) } & 0.0 & 6 & 6 & 25.000 & - & 29.523 & 28.972 & 1.903 & 2.494 \\
\hline & 0.6 & 10 & 9 & 32.746 & 40.618 & 34.330 & 33.884 & 1.317 & 2.494 \\
\hline & 3.0 & 18 & 17 & 42.321 & 46.291 & 45.098 & 44.783 & 0.704 & 1.218 \\
\hline & 10.2 & 32 & 31 & 56.937 & 59.528 & 58.800 & 58.575 & 0.384 & 1.218 \\
\hline & 12.0 & 35 & 34 & 59.641 & 62.105 & 61.274 & 61.060 & 0.350 & 1.218 \\
\hline & 20.1 & 49 & 48 & 69.833 & 71.961 & 72.522 & 72.346 & 0.243 & 1.218 \\
\hline & 29.7 & 63 & 62 & 79.498 & 81.424 & 81.564 & 81.411 & 0.188 & 1.218 \\
\hline
\end{tabular}

Note. - The critical and detection limits, and their associated errors are calculated for (a) $\alpha=\beta=0.1\left(z_{1-\alpha}=z_{1-\beta}=1.282\right)$, (b) $\alpha=0.1, \beta=0.05\left(z_{1-\beta}=1.645\right)$, (c) $\alpha=\beta=0.05$, (d) $z_{1-\alpha}=z_{1-\beta}=3(\alpha=\beta=0.00135)$, (e) $z_{1-\alpha}=3, z_{1-\beta}=5$ $\left(\beta=2.980 \times 10^{-7}\right)$, and (f) $z_{1-\alpha}=z_{1-\beta}=5$. 
Then, the detection limit $\mu_{\mathrm{d}}$ and its estimator are given by, respectively,

$$
\begin{aligned}
& \mu_{\mathrm{d}}=z_{1-\alpha} \sqrt{\mu_{\mathrm{b}}}+z_{1-\beta} \sqrt{\mu_{\mathrm{d}}+\mu_{\mathrm{b}}}, \text { and } \\
& \widehat{S}_{\mathrm{d}}=\frac{\mu_{\mathrm{d}}-z_{1-\alpha} \sqrt{\mu_{\mathrm{b}}}}{\sqrt{\mu_{\mathrm{d}}+\mu_{\mathrm{b}}}} .
\end{aligned}
$$

After some algebraic manipulation, an explicit formula for the detection limit of the source signal may be derived:

$$
\begin{aligned}
\mu_{\mathrm{d}}= & z_{1-\alpha} \sqrt{\mu_{\mathrm{b}}}+\frac{z_{1-\beta}^{2}}{2} \\
& +z_{1-\beta} \sqrt{\mu_{\mathrm{b}}+z_{1-\alpha} \sqrt{\mu_{\mathrm{b}}}+\frac{z_{1-\beta}^{2}}{4}}, \\
\approx & \frac{z_{1-\beta}\left(z_{1-\alpha}+z_{1-\beta}\right)}{2} \\
& +\left(z_{1-\alpha}+z_{1-\beta}\right) \sqrt{\mu_{\mathrm{b}}} \text { for } \mu_{\mathrm{b}} \gg 1, \\
\approx & z_{1-\beta}^{2}+2 z_{1-\alpha} \sqrt{\mu_{\mathrm{b}}} \text { for } \mu_{\mathrm{b}} \ll 1 .
\end{aligned}
$$

In the case of the equal-tailed test $(\alpha=\beta)$, the detection limit becomes

$$
\mu_{\mathrm{d}}=z_{1-\alpha}^{2}+2 z_{1-\alpha} \sqrt{\mu_{\mathrm{b}}} .
$$

Note that this equation is not an approximation and the working formula given in Currie (1968), $\mu_{\mathrm{d}}=2.71+$ $3.29 \sqrt{\mu_{\mathrm{b}}}$, is obtained for the case $\alpha=\beta=0.05$. Using the fact that, for Poisson events, $\mu_{\mathrm{d}}=2.996$ (see Table 1 ), in the case $\mu_{\mathrm{b}}=0$ and $\beta=0.05$, a modification of equation (B8), $\mu_{\mathrm{d}}=3+3.29 \sqrt{\mu_{\mathrm{b}}}$, has been previously proposed for this case (Currie 1972).

It is noticeable that, assuming Gaussian distributions, Bityukov \& Krasnikov (1999) suggested the estimator $\widehat{S}^{*} \equiv \sqrt{\mu_{\mathrm{d}}+\mu_{\mathrm{b}}}-\sqrt{\mu_{\mathrm{b}}}$, the same one as found in this paper for Poisson signals, as a measure of the detection probability in newly planned experiments. They transformed the original Gaussian distributions into standard normal distributions, and the intersection point of the transformed curves was used as a condition to calculate the measure of the detection probability in the context of the equal-probability test. However, the intersection point of the original curves is not at all the same as the intersection point of the transformed curves. With the aid of equation (B8), it can be easily shown that the estimator $\widehat{S}_{\mathrm{d}}$ in equation (B4) is equivalent to their $\widehat{S}^{*}$ when $\alpha=\beta$. Thus, the estimator $\widehat{S}^{*}$ found by Bityukov \& Krasnikov (1999) is, in fact, an estimator of the detection limit in an equal-tailed test, rather than in the equal-probability test as they insisted. 